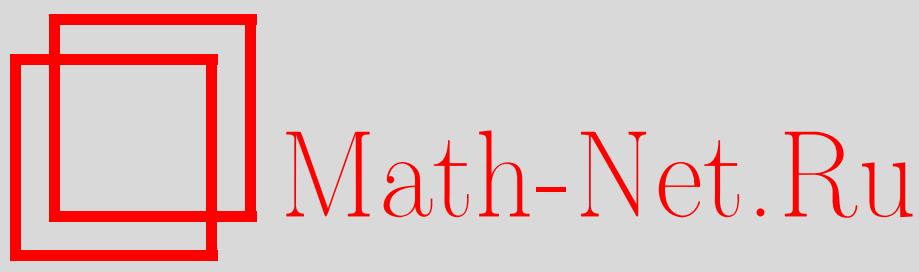

Д. З. Аров, М. А. Нудельман, Признаки подобия всех минимальных пассивных реализаций заданной передаточной функции (матрицы рассеяния или сопротивления), Матем. сб., 2002, том 193, номер 6, 3-24

DOI: https://doi.org/10.4213/sm657

Использование Общероссийского математического портала Math-Net.Ru подразумевает, что вы прочитали и согласны с пользовательским соглашением

http://www . mathnet.ru/rus/agreement

Параметры загрузки:

IP: 3.91 .87 .62

26 апреля 2023 г., 17:14:35 
УДК 517.9

\author{
Д.З. Аров, М.А. Нудельман
}

\title{
Признаки подобия всех минимальных пассивных реализаций заданной передаточной функции (матрицы рассеяния или сопротивления)
}

\begin{abstract}
В работе получены необходимые и достаточные условия на передаточную функцию пассивной линейной стационарной системы рассеяния (сопротивления), при которых минимальные системы указанного класса определяются по передаточной функции с точностью до подобия. Критерии формулируются в терминах ганкелева оператора, символом которого является определяемая по передаточной функции сжимающая оператор-функция, имеющая смысл субоператора внутреннего рассеяния простой консервативной системы рассеяния (соответственно сопротивления) с рассматриваемой передаточной функцией. Прослеживается связь условий подобия с теоремой о короне и ее матричньми обобщениями.

Библиографиял: 23 названия.
\end{abstract}

\section{§1. Введение}

Теория линейных стационарных пассивных (иначе, диссипативных) систем развивается во взаимосвязи с теорией линейных операторов в гильбертовых пространствах и аналитических оператор-функций, теорией рассеяния, корреляционной теорией случайных процессов, теорией управления, теорией пассивных цепей, см. [1]-[8]. После работ Р. Калмана (см. [9]) хорошо известно, что минимальная система (не обязательно пассивная) с конечномерным пространством состояний восстанавливается по своей передаточной функции (п.ф.) с точностью до подобия. Однако для системы с бесконечномерньм пространством состояний это не так: она восстанавливается по п.ф. лиш с точностью до слабого подобия. Оператор слабого подобия может быть неограниченным и неограниченно обратимым, в связи с чем основные операторы слабо подобных систем могут иметь сушественно различные спектральные свойства, а соответствующие им эволюционные полугруппы могут иметь различные асимптотические свойства.

В настоящей работе для минимальных пассивных линейных стационарных систем с передаточными функциями классов Шура и Каратеодори, имеющими смысл

Д. З. Аровьм работа выполнена при частичной финансовой поддержке Гражданского фонда научных исследований и развития США (CRDF US) и Правительства Украины (грант № UM1-2090).

(C) Д. З. Аров, М. А. НУдеЛЬМАН 2002 
матриц рассеяния и сопротивления, установлены необходимые и достаточные условия на п.ф., при которых такие системы определяются по п.ф. с точностью до подобия с ограниченным и ограниченно обратимым оператором подобия. Мы останавливаемся лишь на системах с дискретньм временем. Полученные здесь результаты переносятся на системы с непрерьвньм временем известными преобразованиями, см. [1], [10]. В работе [11] нами получены необходимые и достаточные условия на матрицу рассеяния, при которых все ее минимальные пассивные реализации унитарно подобны. В работе [12] исследовалась проблема подобия минимальных пассивных реализаций матрицы рассеяния. Однако в общем случае она не была решена. Здесь мы тем же методом решаем эту проблему в обшем случае, а также решаем аналогичные проблемы подобия и унитарного подобия для матриц сопротивления. Эти результаты, по сушеству, дают необходимые и достаточные условия на п.ф. класса Шура или Каратеодори, при которых рассматриваемоев теории абсолютной устойчивости систем с нелинейными обратными связями операторное неравенство Калмана-Якубовича-Попова имеет единственное положительное решение $H>0$ (не обязательно ограниченное и ограниченно обратимое), т.е. минимальное и максимальное решения $H_{-}$и $H_{+}$совпадают, а также необходимые и достаточные условия, при которых $H_{+}<\gamma H_{-}$, где $\gamma-$ некоторая константа. Эти приложения будут рассмотрены в совместной работе первого автора с М. А. Каашуком и Д. Р. Пиком.

Авторы благодарят А. А. Нудельмана за помощь при подготовке рукописи.

\section{§ 2. Предварительные сведения}

Все рассматриваемые в работе пространства предполагаются сепарабельньми. Для гильбертовых пространств $U$ и $Y$ через $[U, Y]$ обозначается пространство линейных ограниченных операторов, действующих из $U$ в $Y ;[U]:=[U, U]$. Эволюция линейной стационарной системы с дискретным временем $\Sigma=(A, B, C, D ; X, U, Y)$ описывается системой уравнений

$$
\left\{\begin{aligned}
x(n+1) & =A x(n)+B u(n), \\
y(n) & =C x(n)+D u(n),
\end{aligned}\right.
$$

четырехблочный системный оператор составлен из коэффициентов системы:

$$
M_{\Sigma}:=\left[\begin{array}{cc}
A & B \\
C & D
\end{array}\right] \in[X \oplus U, X \oplus Y]
$$

$x(n) \in X, u(n) \in U$ и $y(n) \in Y$ называются соответственно состоянием, входными и выходным ми данными системы $\Sigma$ в момент времени $n$; гильбертовы пространства $X, U$ и $Y$ называются соответственно пространствами состояний, входных и выходных данных; $A$ называется основным оператором, $\left\{A^{n}\right\}_{n \geqslant 0}$ - эволюиионной полугруппой системъ. Функция

$$
\Theta_{\Sigma}(z)=D+z C(I-z A)^{-1} B
$$


называется передаточной функиией (п.ф.).) системы $\Sigma$, ибо при $x(0)=0$ для $\widehat{u}(z)=$ $\sum_{0}^{\infty} u(n) z^{n}$ и $\widehat{y}(z)=\sum_{0}^{\infty} y(n) z^{n}$ имеем:

$$
\widehat{y}(z)=\Theta_{\Sigma}(z) \widehat{u}(z)
$$

Через $\mathbb{V}_{k} \mathscr{L}_{k}$ обозначается замкнутая линейная оболочка множеств $\mathscr{L}_{k}$,

$$
X_{\Sigma}^{c}=\underset{k \geqslant 0}{\mathbb{V}} A^{k} B U, \quad X_{\Sigma}^{o}=\underset{k \geqslant 0}{\mathbb{V}}\left(A^{*}\right)^{k} C^{*} Y
$$

Система $\Sigma$ называется:

а) управляемой, если $X_{\Sigma}^{c}=X$,

б) наблюдаемой, если $X_{\Sigma}^{o}=X$,

в) простой, если $X_{\Sigma}^{c} \mathbb{V} X_{\Sigma}^{o}=X$,

г) минимальной, если $X_{\Sigma}^{c}=X$ и $X_{\Sigma}^{o}=X$.

Имеется следующее эквивалентное определение минимальности системы, основанное на понятии дилатации.

Система $\widehat{\Sigma}=(\widehat{A}, \widehat{B}, \widehat{C}, D ; \widehat{X}, U, Y)$ называется дилатацией системы $\Sigma=(A, B$, $C, D ; X, U, Y)$, если при некоторых $\mathfrak{D}_{*}$ и $\mathfrak{D}$

$$
\widehat{X}=\mathfrak{D}_{*} \oplus X \oplus \mathfrak{D}, \quad \widehat{A}^{*} \mathfrak{D}_{*} \subset \mathfrak{D}_{*}, \quad \widehat{A} \mathfrak{D} \subset \mathfrak{D}, \quad \widehat{B}^{*} \mathfrak{D}_{*}=\{0\}, \quad \widehat{C} \mathfrak{D}=\{0\}
$$

и

$$
A=P_{X} \widehat{A}\left|X, \quad B=P_{X} \widehat{B}, \quad C=\widehat{C}\right| X \quad(\widehat{D}=D)
$$

где $P_{X}$ - ортопроектор на $X, T \mid X$ - сужение оператора $T$ на $X$. Если $\widehat{\Sigma}$ является дилатацией системы $\Sigma$, то $\Sigma$ называется сужением системы $\widehat{\Sigma}$. Система $\Sigma$ называется минимальной, если она не имеет нетривиального сужения, т.е. если она не является дилатацией никакой другой системы.

Для двух голоморфных функций $\Theta_{1}(z)$ и $\Theta_{2}(z)$ пишем $\Theta_{1} \simeq \Theta_{2}$, если $\Theta_{1}(z) \equiv$ $\Theta_{2}(z)$ в некоторой окрестности точки $z=0$. Если $\widehat{\Sigma}-$ дилатация системы $\Sigma$, то $\Theta_{\widehat{\Sigma}} \simeq \Theta_{\Sigma}$. Если $\Theta \simeq \Theta_{\Sigma}$, то $\Sigma$ назьвается реализацией функиии $\Theta(z)$; если при этом $\Sigma$ - минимальная система, то она называется минимальной реализачией функиии $\Theta$. По произвольной реализации $\Sigma$ функции $\Theta$ можно получить минимальные реализации $\Sigma_{0}$ и $\Sigma_{\bullet}$ этой функции, рассматривая, например, первое и второе сужения $\Sigma_{0}=\Sigma_{\text {rest }, 1}$ и $\Sigma_{\bullet}=\Sigma_{\text {rest }, 2}$ системы $\Sigma$ на подпространства

$$
X_{0}=\overline{P_{X_{\Sigma}^{o}}^{o} X_{\Sigma}^{c}}, \quad X_{\bullet}=\overline{P_{X_{\Sigma}^{c} X_{\Sigma}^{o}}}
$$

где через $\overline{\mathscr{L}}$ обозначается замыкание множества $\mathscr{L}$.

Две системы $\Sigma_{i}=\left(A_{i}, B_{i}, C_{i}, D ; X_{i}, U, Y\right)(i=1,2)$ называются подобными (унитарно подобными), если существует оператор $R \in\left[X_{1}, X_{2}\right]$, имеющий $R^{-1} \in$ $\left[X_{2}, X_{1}\right]$ (соответственно унитарный), такой, что

$$
A_{2}=R A_{1} R^{-1}, \quad B_{2}=R B_{1}, \quad C_{2}=C_{1} R^{-1}
$$

Система $\Sigma$ назьвается пассивной (консервативной) системой рассеяния, если системный оператор $M_{\Sigma}$ является сжимающим (соответственно унитарньм). 
Передаточная функция $\Theta_{\Sigma}$ такой системы называется матрицей рассеяния. Система $\Sigma=(A, B, C, D ; X, U, Y)$ с $Y=U$ назьвается пассивной (консервативной) системой сопротивления, если

$$
\left[\begin{array}{cc}
I-A^{*} A & C^{*}-A^{*} B \\
C-B^{*} A & D+D^{*}-B^{*} B
\end{array}\right] \geqslant 0
$$

(соответственно если $A$ - унитарный оператор, $C=B^{*} A$ и $D+D^{*}=B^{*} B$ ). Передаточная функция такой системы называется матрицей сопротивления. Условие пассивности эквивалентно следующему:

$$
\begin{aligned}
\|u(n)\|^{2}-\|y(n)\|^{2} & \geqslant\|x(n+1)\|^{2}-\|x(n)\|^{2}-\text { для системы рассеяния, } \\
2 \operatorname{Re}(u(n), y(n)) & \geqslant\|x(n+1)\|^{2}-\|x(n)\|^{2}-\text { для системы сопротивления }
\end{aligned}
$$

$((\cdot, \cdot)$ - скалярное произведение в $U(=Y))$, имеющему место для любых $n \geqslant 0$, $x(0) \in X$ и $u(k) \in U(0 \leqslant k \leqslant n)$. В условии консервативности вместо знака " $\geqslant "$ должен стоять знак "=" и таким же свойством должна обладать сопряженная система $\Sigma^{*}=\left(A^{*}, C^{*}, B^{*}, D^{*} ; X, Y, U\right)$.

Пассивная система рассеяния (сопротивления) $\Sigma_{0}$ назьвается оптимальной, если для любой другой пассивной системы рассеяния (соответственно сопротивления) $\Sigma$ с $\Theta_{\Sigma} \simeq \Theta_{\Sigma_{0}}$ для состояний $x_{\Sigma_{0}}(n)$ и $x_{\Sigma}(n)$ этих систем в любой момент времени $n$ при $x_{\Sigma_{0}}(0)=0$ и $x_{\Sigma}(0)=0$ и одинаковых последовательностях входных данных $u(k)(0 \leqslant k \leqslant n)$ имеем

$$
\left\|x_{\Sigma_{0}}(n)\right\| \leqslant\left\|x_{\Sigma}(n)\right\|
$$

Наблюдаемая пассивная система рассеяния (сопротивления) $\Sigma_{\bullet}$ называется *-оптимальной, если для любой другой наблюдаемой пассивной системы рассеяния (соответственно сопротивления) $\Sigma$ с п.ф. $\Theta_{\Sigma} \simeq \Theta_{\Sigma}$ имеем

$$
\left\|x_{\Sigma}(n)\right\| \leqslant\left\|x_{\Sigma_{\bullet}}(n)\right\|
$$

при $x_{\Sigma}(0)=0, x_{\Sigma}(0)=0$ и одинаковой для $\Sigma_{\bullet}$ и $\Sigma$ последовательности $u(k) \in U$ $(0 \leqslant k \leqslant n)$.

Через $\mathscr{S}(U, Y)$ обозначим класс Шура голоморфных в $K=\{z:|z|<1\}$ функций $\Theta(z)$ со значениями из $[U, Y]$ и с $\|\Theta(z)\| \leqslant 1$; через $\mathscr{C}(U)$ обозначим класс Каратеодори голоморфных в $K$ функций $\Theta(z)$ со значениями из $[U]$ и с $\operatorname{Re} \Theta(z) \geqslant 0$.

Справедливы следующие утверждения, см. [1], [13]-[15].

ПРЕДЛОЖЕНИЕ 1. Пусть $\Sigma=(A, B, C, D ; X, U, Y)$ - пассивная система рассеяния (сопротивления).

Тогда $\Theta_{\Sigma} \in \mathscr{S}(U, Y)$ (соответственно $\left.\Theta_{\Sigma} \in \mathscr{C}(U)\right)$.

ПрЕДЛОЖЕНИЕ 2. Пусть $\Theta \in \mathscr{S}(U, Y)(\Theta \in \mathscr{C}(U))$. Тогда существует простая консервативная система рассеяния (соответственно сопротивления) $\Sigma$ с $\Theta_{\Sigma}(z)=\Theta(z)$ и она определяется по $\Theta$ с точностью до унитарного подобия. 
ПРЕДЛОЖЕНИЕ 3. Пусть $\Theta \in \mathscr{S}(U, Y)(\Theta \in \mathscr{C}(U))$. Тогда существуют минимальные оптимальная и *-оптимальная системь рассеяния (соответственно сопротивления) $\Sigma_{0}$ и $\Sigma_{\bullet}$ с п.ф. $\Theta(z)$. Они восстанавливаются по $\Theta c$ точностью до унитарного подобия и могут быть получень соответственно как первое и второе сужения $\Sigma_{0}=\Sigma_{\mathrm{rest}, 1} u \Sigma_{\bullet}=\Sigma_{\mathrm{rest}, 2}$ произвольной консервативной системы рассеяния (сопротивления) $\Sigma$ с $\Theta_{\Sigma}(z)=\Theta(z)$.

Для функции $\Theta \in \mathscr{S}(U, Y)(\Theta \in \mathscr{C}(U))$ будем рассматривать голоморфные в $K$ функции $\varphi(z)$ и $\psi(z)$ со значениями из $[U, \stackrel{\circ}{Y}]$ и $[\stackrel{\circ}{U}, Y](Y=U$ для $\Theta \in \mathscr{C}(U))$, являющиеся решениями факторизационных неравенств (здесь $\stackrel{\circ}{Y}$ и $\stackrel{\circ}{\mathrm{U}}$ - некоторые сепарабельные, возможно, конечномерные гильбертовы пространства):

$$
\text { a) } \varphi^{*}(z) \varphi(z) \leqslant I-\Theta^{*}(z) \Theta(z), \quad \text { б) } \psi(z) \psi^{*}(z) \leqslant I-\Theta(z) \Theta^{*}(z)
$$

соответственно

$$
\text { а) } \varphi^{*}(z) \varphi(z) \leqslant 2 \operatorname{Re} \Theta(z), \text { б) } \psi(z) \psi^{*}(z) \leqslant 2 \operatorname{Re} \Theta(z) \text {, если } \Theta \in \mathscr{C}(U) \text {. }
$$

В множестве решений этих неравенств сушествуют максимальные решения $\varphi_{\Theta}$ и $\psi_{\Theta}$ такие, что для любых других решений имеем

$$
\varphi^{*}(z) \varphi(z) \leqslant \varphi_{\Theta}^{*}(z) \varphi_{\Theta}(z), \quad \psi(z) \psi^{*}(z) \leqslant \psi_{\Theta}(z) \psi_{\Theta}^{*}(z)
$$

Они определяются по $\Theta$ с точностью до постоянных унитарных множителей, слева для $\varphi_{\Theta}$ и справа для $\psi_{\Theta}$, см. [16; гл. V, предложение 4.2] и [13] для $\Theta \in \mathscr{S}(U, Y)$; для $\Theta \in \mathscr{C}(U)$ см. [17]; доказательства аналогичны.

ПреДЛОЖЕНИЕ 4. Пусть $\Theta \in \mathscr{S}(U, Y)(\Theta \in \mathscr{C}(U))$. Пусть $\Sigma$ - простая консервативная система рассеяния (соответственно сопротивления) с п. ф. $\Theta_{\Sigma}(z)=\Theta(z)$. Тогда имеют место следующие эквивалентности:

а) система $\Sigma$ наблюдаема $\Leftrightarrow \varphi_{\Theta}=0$;

б) система $\Sigma$ управляема $\Leftrightarrow \psi_{\Theta}=0$;

в) система $\Sigma$ минимальна $\Leftrightarrow \varphi_{\Theta}=0 u \psi_{\Theta}=0$.

Для системы рассеяния доказательство см. в [14], [15]. Для системы сопротивления доказательство аналогично, см. [17].

TeOpema 1. Пусть $\Theta \in \mathscr{S}(U, Y)(\Theta \in \mathscr{C}(U))$. Пусть maкже $\varphi_{\Theta}=0$ либо $\psi_{\Theta}=0$. Тогда все минимальные пассивные системы рассеяния (сопротивления) с п.ф. $\Theta$ унитарно әквивалентны. Если $\varphi_{\Theta}=0$ и $\psi_{\Theta}=0$, то оник тому же консервативны.

ДокАЗАтЕльство. Пусть $\Sigma$ - простая консервативная система с п.ф. $\Theta(z)$. Тогда $X_{0}=X_{\bullet}=X_{\Sigma}^{c}$, если $\varphi_{\Theta}=0$, и $X_{0}=X_{\bullet}=X_{\Sigma}^{o}$, если $\psi_{\Theta}=0$, где $X_{0}$ и $X_{\bullet}$ определены в $(1)$. Так как при этом минимальные оптимальная и $*$-оптимальная системы $\Sigma_{0}=\Sigma_{\text {rest,1 }}$ и $\Sigma_{\bullet}=\Sigma_{\text {rest,2 }}$ с п.ф. $\Theta(z)$ совпадают, то все минимальные пассивные системы рассеяния (сопротивления) с п.ф. $\Theta(z)$ унитарно подобны [11]. Если $\varphi_{\Theta}=0$ и $\psi_{\Theta}=0$, то $\Sigma_{0}=\Sigma_{\bullet}=\Sigma$ и потому все минимальные пассивные системы с п.ф. $\Theta$ консервативны. 


\section{§3. Субоператор внутреннего рассеяния $s_{\Theta}(\zeta)$ и соответствующий ганкелев оператор $\Gamma_{\Theta}$}

3.1. Определение субоператора внутреннего рассеяния. Исследуем теперь проблемы подобия и унитарного подобия всех минимальных пассивных реализаций заданной матрицы рассеяния $\Theta(\in \mathscr{S}(U, Y))$ или матрицы сопротивления $\Theta$ $(\in \mathscr{C}(U))$ в случае, когда $\varphi_{\Theta} \neq 0$ и $\psi_{\Theta} \neq 0$.

Пусть $\Sigma=(A, B, C, D ; X, U, Y)$ - простая консервативная система рассеяния или сопротивления с п.ф. $\Theta$. Пусть $\varphi_{\Theta} \neq 0$ и $\psi_{\Theta} \neq 0$. Это означает, что система $\Sigma$ не является ни наблюдаемой, ни управляемой. Рассмотрим пространства

$$
\mathfrak{D}_{-}=X \ominus X_{\Sigma}^{c}, \quad \mathfrak{D}_{+}=X \ominus X_{\Sigma}^{o}
$$

Имеем: $A^{*} \mathfrak{D}_{-} \subset \mathfrak{D}_{-}, A \mathfrak{D}_{+} \subset \mathfrak{D}_{+}$. Более того, операторы

$$
V_{-}:=A^{*} \mid \mathfrak{D}_{-}\left(\in\left[\mathfrak{D}_{-}\right]\right) \text {и } V_{+}:=A \mid \mathfrak{D}_{+}\left(\in\left[\mathfrak{D}_{+}\right]\right)
$$

- простые полуунитарные, т.е. изометрически отображают соответственно $\mathfrak{D}_{-}$ и $\mathfrak{D}_{+}$в себя и не имеют унитарных частей. Если для унитарного (сжимаюшего) оператора $A(\in[X])$ и простых полуунитарных операторов $V_{ \pm}\left(\in\left[\mathfrak{D}_{ \pm}\right]\right)$имеют место соотношения (5), то говорят, что $A$ является унитарным (сәмимающим) сцеплением простых полуунитарных операторов $V_{-}$и $V_{+}$в пространстве $X$, и для этого сцепления вводятся понятия оператора рассеяния и субоператора рассеяния. Для унитарных сцеплений это было сделано в работе [18] в порядке обобшения консервативной схемы рассеяния Лакса-Филлипса с дискретным временем, а для сжимающих сцеплений - в работе [19] в порядке обобщения диссипативной схемы рассеяния Лакса-Филлипса с дискретньм временем. Следуя, в основном, работе [18], введем понятия оператора рассеяния и субоператора рассеяния унитарного сцепления $A$ простых полуунитарных операторов $V_{-}$и $V_{+} \cdot$ Для этого рассмотрим дефектные подпространства $\mathfrak{N}_{-}$и $\mathfrak{N}_{+}$операторов $V_{-}$и $V_{+}$:

$$
\mathfrak{N}_{-}=\mathfrak{D}_{-} \ominus V_{-} \mathfrak{D}_{-}, \quad \mathfrak{N}_{+}=\mathfrak{D}_{+} \ominus V_{+} \mathfrak{D}_{+} .
$$

Рассмотрим также подпространства

$$
X_{-}=\underset{n \geqslant 0}{\mathbb{V}} A^{n} \mathfrak{D}_{-}, \quad X_{+}=\underset{n \geqslant 0}{\mathbb{V}}\left(A^{*}\right)^{n} \mathfrak{D}_{+} .
$$

Справедливы разложения Вольда:

$$
\begin{aligned}
\mathfrak{D}_{-}=\bigoplus_{-\infty}^{0} A^{n} \mathfrak{N}_{-}, & X_{-}=\bigoplus_{-\infty}^{+\infty} A^{n} \mathfrak{N}_{-}, \\
\mathfrak{D}_{+}=\bigoplus_{0}^{+\infty} A^{n} \mathfrak{N}_{+}, & X_{+}=\bigoplus_{-\infty}^{+\infty} A^{n} \mathfrak{N}_{+},
\end{aligned}
$$


и для любых $x \in X$ имеем

$$
P_{X_{ \pm}} x=\bigoplus_{-\infty}^{+\infty} P_{A^{k} \mathfrak{N}_{ \pm}} x=\bigoplus_{-\infty}^{+\infty} A^{k} P_{\mathfrak{N}_{ \pm}} A^{-k} x
$$

Пусть $L^{2}(\mathfrak{N})$ - гильбертово пространство слабо измеримых на $\partial K$ функций $h(\zeta)$ $\left(=\sum_{-\infty}^{+\infty} h_{k} \zeta^{k}\right)$ со значениями из гильбертова пространства $\mathfrak{N}$ и с

$$
\begin{gathered}
\|h\|^{2}=\frac{1}{2 \pi} \int_{\partial K}\|h(\zeta)\|^{2}|d \zeta|\left(=\sum_{-\infty}^{+\infty}\left\|h_{k}\right\|^{2}\right)<\infty \\
H^{2}(\mathfrak{N})=\left\{h(\zeta) \in L^{2}(\mathfrak{N}): h(\zeta)=\sum_{0}^{\infty} h_{k} \zeta^{k}\right\} \\
K^{2}(\mathfrak{N})=\left\{h(\zeta) \in L^{2}(\mathfrak{N}): h(\zeta)=\sum_{-\infty}^{1} h_{k} \zeta^{k}\right\}
\end{gathered}
$$

$H^{2}(\mathfrak{N})$ отождествляется с пространством Харди голоморфных в $K$ функций $h(z)=\sum_{0}^{\infty} h_{k} z^{k}$ со значениями из гильбертова пространства $\mathfrak{N}$ и с $\|h\|^{2}=$ $\sum_{0}^{\infty}\left\|h_{k}\right\|^{2}<\infty$ путем отождествления $h(z)$ с радиальным граничным значением $h(\zeta)$. Рассмотрим обобщенные преобразования Фурье $\mathscr{F}_{ \pm}\left(\in\left[X, L^{2}\left(\mathfrak{N}_{ \pm}\right)\right]\right)$, определяемые по формулам

$$
\mathscr{F}_{-} x=\sum_{-\infty}^{+\infty}\left(P_{\mathfrak{N}_{-}} A^{k} x\right) \zeta^{k}, \quad \mathscr{F}_{+} x=\sum_{-\infty}^{+\infty}\left(P_{\mathfrak{N}_{+}} A^{k} x\right) \zeta^{k-1}, \quad x \in X
$$

Они обладают следующими свойствами:

а) $\mathscr{F}_{ \pm}$изометрически отображают $X_{ \pm}$на $L^{2}\left(\mathfrak{N}_{ \pm}\right)$;

б) $\operatorname{Ker} \mathscr{F}_{ \pm}=X \ominus X_{ \pm}$;

в) $\mathscr{F}_{-} \mathfrak{D}_{-}=H^{2}\left(\mathfrak{N}_{-}\right), \mathscr{F}_{+} \mathfrak{D}_{+}=K^{2}\left(\mathfrak{N}_{+}\right)$;

г) $\left(\mathscr{F}_{ \pm} A x\right)(\zeta)=\zeta^{-1}\left(\mathscr{F}_{ \pm} x\right)(\zeta), x \in X$

Оператор

$$
S=\mathscr{F}_{+} \mathscr{F}_{-}^{*}\left(\in\left[L^{2}\left(\mathfrak{N}_{-}\right), L^{2}\left(\mathfrak{N}_{+}\right)\right]\right)
$$

называется оператором рассеяния унитарного сцепления A простых полуунитарных операторов $V_{-} u V_{+}$. Так как $\|S\| \leqslant 1$ и $S$ сплетает унитарные операторы умножения на $\zeta$ соответственно в пространствах $L^{2}\left(\mathfrak{N}_{-}\right)$и $L^{2}\left(\mathfrak{N}_{+}\right)$, то существует единственная слабо измеримая в существенном ограниченная на $\partial K$ сжимающая функция $s(\zeta)$ со значениями из $\left[\mathfrak{N}_{-}, \mathfrak{N}_{+}\right]\left(s(\zeta) \in L^{\infty}\left(\mathfrak{N}_{-}, \mathfrak{N}_{+}\right),\|s(\cdot)\|_{\infty} \leqslant 1\right)$ такая, что

$$
(S h)(\zeta)=s(\zeta) h(\zeta) \text { п.в., } h \in L^{2}\left(\mathfrak{N}_{-}\right)
$$

Функция $s(\zeta)$ называется субоператором рассеяния унитарного сцепления $A$ простых полуунитарных операторов $V_{-} u V_{+}[18]$. 
В работе [18] также показано, что если $E_{\mu}$ - спектральная функция рассматриваемого унитарного оператора $A\left(A=\int_{-\pi}^{\pi} e^{-i \mu} d E_{\mu}\right)$, то функции $\sigma(\mu ; x)=$ $\left(E_{\mu} x, x\right)$ являются абсолютно непрерывными на $[-\pi, \pi]$ для $x \in X_{-} \mathbb{V} X_{+}$, а для субоператора рассеяния $s(\zeta)$ справедлива формула

$$
s\left(e^{i \mu}\right)=2 \pi e^{-i \mu} \frac{1}{d \mu} P_{\mathfrak{N}_{+}} E_{\mu} \mid \mathfrak{N}_{-} \text {п.в. }
$$

где производная от оператор-функции рассматривается в слабом смысле. Из этой формулы видно, что субоператор рассеяния $s\left(e^{i \mu}\right)$ зависит лишь от части $A \mid X_{-} \mathbb{V} X_{+}$оператора $A$.

Если $A(\in[X])$ - сжимающее сцепление простых полуунитарных операторов, то вместо $A$ можно рассмотреть минимальную унитарную дилатацию $\widehat{A}(\in[\widehat{X}])$ оператора $A$. Оператор $\widehat{A}$ является унитарньм сцеплением тех же простых полуунитарных операторов $V_{-}$и $V_{+}$в пространство $\widehat{X}(\supset X)$. Оператором рассеяния $S$ и субоператором рассеяния $s(\zeta)$ сжимаюшего сцепления $A$ простых полуунитарных операторов $V_{-}$и $V_{+}$в пространство $X$ являются оператор рассеяния и субоператор рассеяния унитарного сцепления $\widehat{A}$ тех же операторов $V_{-}$и $V_{+}$в пространство $\widehat{X}$, см. [16].

Если $\mathfrak{D}_{-}$и $\mathfrak{D}_{+}$ортогональны в $X$, то в этом и только этом случае $s(\zeta)$ является радиальным граничным значением некоторой функции $s(z)$ класса $\mathscr{S}\left(\mathfrak{N}_{-}, \mathfrak{N}_{+}\right)$,

$$
s(\zeta)=s-\lim _{r \uparrow 1} s(r \zeta) \text { п.в., }|\zeta|=1 .
$$

В этом случае сцепление $A$ называется ортогональным, а функция $s(z)$ называется матрицей рассеяния ортогонального сцепления $A$ операторов $V_{-} u V_{+}$ в пространство $X\left(\supset \mathfrak{D}_{-} \oplus \mathfrak{D}_{+}\right)$. Из формулы Коши и формулы $(10)$ вытекает следующая формула для матрицы рассеяния:

$$
S(z)=P_{\mathfrak{N}_{+}} A(I-z A)^{-1} \mid \mathfrak{N}_{-} .
$$

Поскольку оператор рассеяния $S$ и субоператор рассеяния $s(\zeta)$ нами построены вполне определенным образом по простой консервативной системе сопротивления или рассеяния $\Sigma$ с п.ф. $\Theta(z)$ (класса $\mathscr{C}(U)$ или $\mathscr{S}(U, Y)$ соответственно), а $\Sigma$ определяется по п.ф্. $\Theta(z)$ с точностью до унитарной эквивалентности, то $S$ и $s(\zeta)$ в сушественном однозначно определяются по $\Theta(z)$. Точнее, $s(\zeta)$ определяется по $\Theta(z)$ с точностью до умножения слева и справа на постоянные унитарные операторы $s(\zeta) \rightarrow R_{1} s(\zeta) R_{2}$, где $R_{1}\left(\in\left[\mathfrak{N}_{+}, Y_{0}\right]\right)$ и $R_{2}\left(\in\left[U_{0}, \mathfrak{N}_{-}\right]\right)$- некоторые унитарные операторы. Учитывая это, будем пользоваться обозначениями:

$$
S_{\Theta}=S, \quad s_{\Theta}(\zeta)=s(\zeta), \quad s_{\Theta}(z)=s(z)
$$

Будем называть $S_{\Theta}, s_{\Theta}(\zeta)$ и $s_{\Theta}(z)$ соответственно оператором внутреннего рассеяния, субоператором внутреннего рассеяния и матрицей внутреннего рассеяния простой консервативной системы $\Sigma$ с п.ф. $\Theta(z)$ (класса $\mathscr{C}(U)$ или $\mathscr{S}(U, Y))$. Выделенные в пространстве состояний $X$ системы $\Sigma$ подпространства $\mathfrak{D}_{-}$и $\mathfrak{D}_{+}$и простые полуунитарные операторы в них $V_{-}$и $V_{+}$интерпретируются как внутренние каналы рассеяния для системы $\Sigma$. 
3.2. Аналитическое определение функции $s_{\Theta}(\zeta)$. Идея выделения внутренних каналов рассеяния у простой консервативной системы рассеяния возникла и сушественно использовалась в исследованиях первого автора при развитии метода Дарлингтона [14], [17]. На этом пути в [14] была введена в рассмотрение функция $s_{\Theta}(\zeta)$ для $\Theta \in \mathscr{S}(U, Y)$, однако только в случае, когда разрешимы факторизационные уравнения

$$
\begin{aligned}
& \text { а) } \varphi^{*}(\zeta) \varphi(\zeta)=I-\Theta^{*}(\zeta) \Theta(\zeta) \text { п.в., } \varphi \in \mathscr{S}\left(U, Y_{0}\right) \\
& \text { б) } \psi(\zeta) \psi^{*}(\zeta)=I-\Theta(\zeta) \Theta^{*}(\zeta) \text { п.в., } \psi \in \mathscr{S}\left(U_{0}, Y\right) .
\end{aligned}
$$

При этом $\varphi=\varphi_{\Theta} \in \mathscr{S}\left(U, Y_{0}\right)$ и $\psi=\psi_{\Theta} \in \mathscr{S}\left(U_{0}, Y\right)$ являются максимальными решениями этих уравнений, а $s_{\Theta}(\zeta)$ может быть определена как единственная функция $s(\zeta)$ из $L^{\infty}\left(U_{0}, Y_{0}\right)$ такая, что функция

$$
\widetilde{s}_{\Theta}(\zeta):=\left[\begin{array}{cc}
\psi_{\Theta}(\zeta) & \Theta(\zeta) \\
s(\zeta) & \varphi_{\Theta}(\zeta)
\end{array}\right] \quad\left(\in L^{\infty}\left(U_{0} \oplus U, Y \oplus Y_{0}\right)\right)
$$

является унитарнозначной п.в. на $\partial K$. Это условие равносильно тому, что $\varphi_{\Theta}$ и $\psi_{\Theta}$ являются решениями задач $(12)$, и определению функции $s_{\Theta}(\zeta)$ соотношением

$$
s_{\Theta}(\zeta) \psi_{\Theta}^{*}(\zeta)=-\varphi_{\Theta}(\zeta) \Theta^{*}(\zeta) \text { п.в. }
$$

В обшем случае для $\Theta(z) \in \mathscr{S}(U, Y)$ функция $s_{\Theta}(\zeta)$ введена в работе [20] указанньм выше образом как субоператор рассеяния унитарного сцепления $\widehat{A}(\in[\widehat{X}])$ простых полуунитарных операторов $V_{-}$и $V_{+}$. Там же показано, что $s(\zeta)=s_{\Theta}(\zeta)$ является единственным решением следуюшей аналитической задачи. Пусть $\Theta \in$ $\mathscr{S}(U, Y)$. Далее, пусть $\varphi_{\Theta} \in \mathscr{S}\left(U, Y_{0}\right)$ и $\psi_{\Theta} \in \mathscr{S}\left(U_{0}, Y\right)$ - максимальные решения факторизационных неравенств (2). Требуется найти функцию $s(\zeta)$ такую, что

$$
\widetilde{s}(\zeta)=\left[\begin{array}{cc}
\psi_{\Theta}(\zeta) & \Theta(\zeta) \\
s(\zeta) & \varphi_{\Theta}(\zeta)
\end{array}\right] \in L^{\infty}\left(U_{0} \oplus U, Y \oplus Y_{0}\right), \quad\|\widetilde{s}\|_{\infty} \leqslant 1
$$

$\mathrm{K}$ сожалению, для $\Theta$ класса $\mathscr{C}(U)$ мы не имеем аналогичного определения функции $s_{\Theta}(\zeta)$ в общем случае. Однако в случае, когда существует решение $\varphi(z) \in$ $H^{2}(U, \stackrel{\circ}{Y})$ или $\psi^{*}(\bar{z}) \in H^{2}(Y, \stackrel{\circ}{U})$ одного из факторизационных уравнений

$$
\begin{array}{ll}
\text { а) } \varphi^{*}(\zeta) \varphi(\zeta)=2 \operatorname{Re} \Theta(\zeta) \text { п.в. }|\zeta|=1, \\
\text { б) } \psi(\zeta) \psi^{*}(\zeta)=2 \operatorname{Re} \Theta(\zeta) \text { п.в. }|\zeta|=1,
\end{array}
$$

имеется следующий аналог формулы (14).

Факторизационные задачи (16) являются аналогами задач (12). В случае, когда $\operatorname{dim} U=\infty$, их следует понимать в следуюшем смысле. Включение $\varphi(z) \in$ $H^{2}(U, \stackrel{\circ}{Y})$ означает, что $\varphi(z)$ является голоморфной в $K$ функцией со значениями из $[U, \stackrel{\circ}{Y}]$ такой, что $\varphi(z) u \in H^{2}(\stackrel{\circ}{Y})$ при любом $u \in U$. Соотношение а) в (16) означает, что

$$
\lim _{r \uparrow 1}\|\varphi(r \zeta) u\|^{2}=\lim _{r \uparrow 1} \operatorname{Re}(\Theta(r \zeta) u, u) \quad \text { п.в., } \quad \forall u \in U .
$$


Аналогичный смысл имеет и решение $\psi(z)$ задачи б) в (16):

$$
\lim _{r \uparrow 1}\left\|\psi^{*}(r \zeta) u\right\|^{2}=\lim _{r \uparrow 1} \operatorname{Re}(\Theta(r \zeta) u, u) \quad \text { п.в., } \quad \forall u \in U .
$$

В случае, когда разрешима задача а) в (16) (соответственно б)), функция $\varphi_{\Theta}(z)$ (соответственно $\left.\psi_{\Theta}(z)\right)$ является максимальньм решением этой задачи. Как будет показано, в этом случае функция $s_{\Theta}(\zeta)$ определяется соотношением соответственно

$$
\begin{aligned}
& \text { а) } s_{\Theta}^{*}(\zeta) \varphi_{\Theta}(\zeta)=\psi_{\Theta}^{*}(\zeta) \text { п.в., } \\
& \text { б) } s_{\Theta}(\zeta) \psi_{\Theta}^{*}(\zeta)=\varphi_{\Theta}(\zeta) \text { п.в. }
\end{aligned}
$$

ПРЕДЛОЖЕНИЕ 5. Пусть $\Theta \in \mathscr{C}(U)$ u $\Sigma=(A, B, C, D ; X, U, U)-$ nростая консервативная система сопротивления с матрицей сопротивления $\Theta(z)$. Пусть $\mathfrak{N}_{+}$u $\mathfrak{N}_{-}-$подпространства, определяемые по формулам (4), (6). Тогда функиии $\varphi_{\Theta}(z) \in H^{2}\left(U, \mathfrak{N}_{+}\right)$и $\psi_{\Theta}^{*}(\bar{z}) \in H^{2}\left(Y, \mathfrak{N}_{-}\right)$, определяемие по формулам
a) $\varphi_{\Theta}(z)=P_{\mathfrak{N}_{+}}(I-z A)^{-1} B$
б) $\psi_{\Theta}^{*}(\bar{z})=P_{\mathfrak{N}_{-}}\left(I-z A^{*}\right)^{-1} A^{*} B$,

являются максимальнылми решениями неравенств а) и б) в (3) и
a) $\varphi_{\Theta}(\zeta) u=\left(\mathscr{F}+A^{*} B u\right)(\zeta)$,
б) $\psi_{\Theta}^{*}(\zeta) u=\left(\mathscr{F}-A^{*} B u\right)(\zeta)$,

где $\mathscr{F}_{+}$и $\mathscr{F}_{-}-$обобщенные преобразования Фурье, определенные формула$\mathcal{M u}(7)$.

Доказательство. Пусть $\Theta \in \mathscr{C}(U), \Sigma=(A, B, C, D ; X, U, U)$ - простая консервативная система сопротивления с $\Theta_{\Sigma}(z)=\Theta(z)$. Рассмотрим $\Sigma_{0}=\Sigma_{\text {rest }, 1}=$ $\left(A_{0}, B_{0}, C_{0}, D ; X_{0}, U, U\right)$ - минимальную и оптимальную систему сопротивления, получаемую как первое сужение системы $\Sigma$. Положим

$$
N_{0}=P_{\mathfrak{N}_{+}} B, \quad M_{0}=P_{\mathfrak{N}_{+}} A
$$

Так как

$$
B U \subset X_{0} \oplus \mathfrak{N}_{+}, \quad A X_{0} \subset X_{0} \oplus \mathfrak{N}_{+},
$$

то получим равенство

$$
\left[\begin{array}{cc}
I-A_{0}^{*} A_{0} & C_{0}^{*}-A_{0}^{*} B_{0} \\
C_{0}-B_{0}^{*} A_{0} & D+D^{*}-B_{0}^{*} B_{0}
\end{array}\right]=\left[\begin{array}{c}
M_{0}^{*} \\
N_{0}^{*}
\end{array}\right]\left[M_{0}, N_{0}\right]
$$

Пользуясь этим равенством, получаем тождество

$$
\Theta(z)+\Theta^{*}(z)=\left(1-|z|^{2}\right) B_{0}^{*}\left(I-\bar{z} A_{0}^{*}\right)^{-1}\left(I-z A_{0}\right)^{-1} B_{0}+\varphi_{\Sigma_{0}}^{*}(z) \varphi_{\Sigma_{0}}(z),
$$

где

$$
\begin{aligned}
\varphi_{\Sigma_{0}}(z) & =N_{0}+z M_{0}\left(I-z A_{0}\right)^{-1} B_{0}=P_{\mathfrak{N}_{+}}\left(B+z A\left(I-z A_{0}\right)^{-1} B_{0}\right) \\
& =P_{\mathfrak{N}_{+}}\left(B+z A(I-z A)^{-1} B\right)=P_{\mathfrak{N}_{+}}(I-z A)^{-1} B .
\end{aligned}
$$


Повторяя рассуждения из статьи [14], проведенные для $\Theta \in \mathscr{S}(U, Y)$, можно доказать, что из соотношения (20) и того факта, что $\Sigma_{0}$ - оптимальная система сопротивления с п.ф. $\Theta(z)$, вытекает, что $\varphi_{\Sigma_{0}}(z)$ - максимальное решение факторизационного неравенства а) в $(3)$, т.е. что $\varphi_{\Theta}(z)=\varphi_{\Sigma_{0}}(z)$. Тем самьм доказана формула а) в (18). Пользуясь ею, получаем, что

$$
\varphi_{\Theta}(\zeta) u=\sum_{0}^{\infty}\left(P_{\mathfrak{N}_{+}} A^{k} B u\right) \zeta^{k}
$$

В то же время, так как $A^{*} B U \subset X_{\Sigma}^{o}$, то

$$
\begin{gathered}
P_{X_{+}} A^{*} B u=\bigoplus_{1}^{\infty} A^{-k} P_{\mathfrak{N}_{+}} A^{k-1} B u \\
\left(\mathscr{F}_{+} A^{*} B u\right)(\zeta)=\sum_{0}^{\infty}\left(P_{\mathfrak{N}_{+}} A^{k} B u\right) \zeta^{k}=\varphi_{\Theta}(\zeta) u
\end{gathered}
$$

т.е. справедлива формула а) в (19).

Поскольку $\psi_{\Theta}^{*}(\bar{z})$ определяется по $\Theta^{*}(\bar{z})$ точно так же, как $\varphi_{\Theta}(z)$ определяется по $\Theta(z)$, а $\Theta^{*}(\bar{z})$ является п.ф. . простой консервативной системы сопротивления $\Sigma^{*}$ и $X_{\Sigma^{*}}^{c}=X_{\Sigma}^{o}, X_{\Sigma^{*}}^{o}=X_{\Sigma}^{c}$, то согласно уже доказанному для $\varphi_{\Theta}(z)$ получаем, что

$$
\psi_{\Theta}^{*}(\bar{z})=P_{\mathfrak{N}_{-}^{*}}\left(I-z A^{*}\right)^{-1} C^{*}=P_{\mathfrak{N}_{-}}\left(I-z A^{*}\right)^{-1} A^{*} B,
$$

т.е. справедлива формула б) в (18). Кроме того,

$$
\psi_{\Theta}^{*}(\zeta) u=\sum_{0}^{\infty}\left(P_{\mathfrak{N}_{-}} A^{* k+1} B u\right) \zeta^{-k}=\left(\mathscr{F}_{-} A^{*} B u\right)(\zeta),
$$

так что справедлива формула б) в (19).

ПрЕДЛОЖЕНИЕ 6. Пусть $\Theta \in \mathscr{C}(U)$. Пусть для $\Theta$ разрешимо одно из факторизачионных уравнений а) или б) в (17). Пусть $\varphi_{\Theta}$ и $\psi_{\Theta}$ - максимальные решения факторизационных неравенств (3). Тогда субоператор внутреннего рассеяния $s_{\Theta}(\zeta)$ мохет быть определен соответственно соотношением а) или б) в (17).

ДоказАТЕЛьство. Не ограничивая общности, полагаем $X=X_{-} \mathbb{V} X_{+}$. Пусть разрешимо уравнение б) в (17). Тогда

$$
\begin{gathered}
\left(\mathscr{F}_{-} \sum_{-m}^{n} A^{k} B u_{k}\right)(\zeta)=\psi_{\Theta}^{*}(\zeta) h(\zeta), \quad \text { где } h(\zeta)=\sum_{-m}^{n} u_{k} \zeta^{-k+1}, \\
\left\|\mathscr{F}_{-} \sum_{-m}^{n} A^{k} B u_{k}\right\|_{L^{2}\left(\mathfrak{N}_{-}\right)}^{2} \\
=\left\|\psi_{\Theta}^{*}(\zeta) h(\zeta)\right\|_{L^{2}\left(\mathfrak{N}_{-}\right)}^{2} \\
=2 \frac{1}{2 \pi} \int_{-\pi}^{\pi} \operatorname{Re}(\Theta(\zeta) h(\zeta), h(\zeta))|d \zeta|=\left\|\sum_{-m}^{n} A^{k} B u_{k}\right\|^{2} .
\end{gathered}
$$


Здесь использовано предположение, что $X_{-} \mathbb{V} X_{+}=X$, из которого следует, что унитарный оператор $A$ имеет абсолютно непрерывный спектр, т.е. что функции $\sigma(\mu, x)=\left(E_{\mu} x, x\right)$ являются абсолютно непрерывньми для всех $x \in X$. Так как $\mathbb{V}_{-\infty}^{\infty} A^{k} B U=X$, то получаем, что $\mathscr{F}-$ изометрично на всем $X$, т.е. $X{ }_{-}=X$ и, следовательно, $X_{+} \subset X_{-}$. Мы имеем:

$$
\mathscr{F}_{+}\left(A^{*} B u\right)=\varphi_{\Theta}(\zeta) u, \quad \mathscr{F}_{-}\left(A^{*} B u\right)=\psi_{\Theta}^{*}(\zeta) u
$$

и $\mathscr{F}_{-}\left(\in\left[X, L^{2}\left(\mathfrak{N}_{-}\right)\right]\right)$- унитарный оператор. Поэтому

$$
\left(\mathscr{F}_{+} \mathscr{F}_{-}^{*}\right)\left(\psi_{\Theta}^{*}(\zeta) u\right)=\mathscr{F}_{+}\left(A^{*} B u\right)=\varphi_{\Theta}(\zeta) u .
$$

Осталось учесть, что $s_{\Theta}(\zeta)=s(\zeta)$ определяется по формулам $(8),(9)$. В итоге приходим к соотношению б) в (17). Этим соотношением функция $s_{\Theta}(\zeta)$ определяется однозначно (как элемент пространства $L^{\infty}\left(U_{0}, Y_{0}\right)$ ), ибо из него вытекает, что

$$
s_{\Theta}(\bar{\zeta}) \psi_{\Theta}^{*}(\bar{\zeta}) h(\zeta)=\varphi_{\Theta}(\bar{\zeta}) h(\zeta), \quad h(\zeta)=\sum_{-m}^{n} u_{k} \zeta^{k} \in L^{2}(U)
$$

Так как $\psi_{\Theta}^{*}(\bar{z})$ - внешняя функция, то

$$
\underset{-\infty}{\mathbb{V}} \psi_{\Theta}^{*}(\bar{\zeta}) \zeta^{k} U=L^{2}\left(U_{0}\right)
$$

и потому сжимаюший оператор умножения на $s_{\Theta}(\bar{\zeta})$, действующий из $L^{2}\left(U_{0}\right)$ в $L^{2}\left(Y_{0}\right)$, однозначно определен соотношением б) в (17).

Если $\varphi_{\Theta}(\zeta)$ является решением факторизационной задачи а) в (12), то в предположении, что $X_{-} \mathbb{v} X_{+}=X$, получим равенство $X_{+}=X$, равносильное тому, что $\mathscr{F}_{+}\left(\in\left[X, L^{2}\left(\mathfrak{N}_{+}\right)\right]\right)-$унитарный оператор. При этом $X_{-} \subset X_{+}=X$ и

$$
\left(\mathscr{F}_{-} \mathscr{F}_{+}^{*}\right) \varphi_{\Theta}(\zeta) u=\psi_{\Theta}^{*}(\zeta) u,
$$

T.e.

$$
s_{\Theta}^{*}(\zeta) \varphi_{\Theta}(\zeta) u=\psi_{\Theta}^{*}(\zeta) u \text { п.в., } u \in U .
$$

Пришли к соотношению а) в (17). Этим соотношением однозначно определяется функция $s_{\Theta}^{*}(\zeta)$, а значит, и $s_{\Theta}(\zeta)\left(\in L^{\infty}\left(U_{0}, Y_{0}\right)\right)$ : так как $\varphi_{\Theta}(z)-$ внешняя функция, то

$$
\underset{-\infty}{\mathbb{V}} \varphi_{\Theta}(\zeta) \zeta^{k} U=L^{2}\left(Y_{0}\right),
$$

и потому сжимающий оператор умножения на $s_{\Theta}^{*}(\zeta)$, действующий из $L^{2}\left(Y_{0}\right)$ в $L^{2}\left(U_{0}\right)$, однозначно определен соотношением а) в (17).

Заметим, что если обе задачи а) и б) в (18) разрешимы, то $X_{-}=X_{+}=X$. При этом $s_{\Theta}(\zeta)$ - унитарнозначная функция п.в. на $\partial K$, определяемая каждым из соотношений а) и б) в (17). 
3.3. Ганкелев оператор $\Gamma_{\Theta}$ с символом $s_{\Theta}$, его геометрический смысл. Пусть $\Theta$ принадлежит классу $\mathscr{S}(U, Y)$ или $\mathscr{C}(U)$. Пусть $s_{\Theta}(\zeta)$ - соответствуюший субоператор внутреннего рассеяния, отвечающий функции $\Theta(z)$ согласно п. 3.1. Пусть $\Sigma$ - простая консервативная система (соответственно рассеяния или сопротивления) с п.ф. $\Theta(z)$. Пусть $V_{ \pm}\left(\in\left[\mathfrak{D}_{ \pm}\right]\right)$- рассмотренные в п. 3.1 простые полуунитарные операторы. Рассмотрим оператор

$$
\Gamma=P_{\mathfrak{D}_{+}} \mid \mathfrak{D}_{-},
$$

определяющий угол между подпространствами $\mathfrak{D}_{-}$и $\mathfrak{D}_{+}$. Рассмотрим также ганкелев оператор $\Gamma_{\Theta}$ с символом $s_{\Theta}(\zeta)$ :

$$
\Gamma_{\Theta}=P_{K^{2}\left(\mathfrak{N}_{+}\right)} M_{s_{\Theta}} \mid H^{2}\left(\mathfrak{N}_{-}\right)
$$

где $M_{s_{\Theta}}\left(\in\left[L^{2}\left(\mathfrak{N}_{-}\right), L^{2}\left(\mathfrak{N}_{+}\right)\right]\right)$- оператор умножения на $s_{\Theta}(\zeta)$, т.е. $M_{s_{\Theta}}=S_{\Theta}-$ оператор внутреннего рассеяния. Имеем:

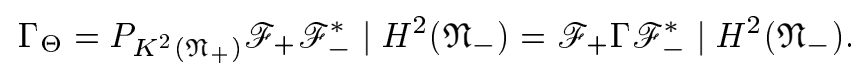

Здесь использованы свойства а)-г) преобразований $\mathscr{F}_{ \pm}$. Учитывая, что $\mathscr{F}+$ изометрически отображает $\mathfrak{D}_{+}$на $K^{2}\left(\mathfrak{N}_{+}\right)$, а $\mathscr{F}_{-}$изометрически отображает $\mathfrak{D}_{-}$на $H^{2}\left(\mathfrak{N}_{-}\right)$, получаем, что операторы $Г$ и $\Gamma_{\Theta}$ связаны соотношениями

$$
\Gamma_{\Theta}=\mathscr{F}_{+} \Gamma \mathscr{F}_{-}^{*}\left|H^{2}\left(\mathfrak{N}_{-}\right), \quad \Gamma=\mathscr{F}_{+}^{*} \Gamma_{\Theta} \mathscr{F}_{-}\right| \mathfrak{D}_{-} .
$$

\section{§4. Основные результаты}

Теперь мы может сформулировать и доказать основные результаты работы.

Пусть $\Theta$ - функция класса $\mathscr{S}(U, Y)$ либо $\mathscr{C}(U)$. Если для нее $\varphi_{\Theta}=0$ (т.е. $U_{0}=\mathfrak{N}_{-}=\{0\}$ ) либо $\psi_{\Theta}=0$ (т.е. $Y_{0}=\mathfrak{N}_{+}=\{0\}$ ), то будем считать, что $s_{\Theta}(\zeta)=0$ и $\Gamma_{\Theta}=0$.

Teopema 2. Пусть $\Theta \in \mathscr{S}(U, Y)$ либо $\Theta \in \mathscr{C}(U)$. Пусть $s_{\Theta}(\zeta)\left(\in L^{\infty}\left(U_{0}, Y_{0}\right)\right)$ - соответствующий субоператор внутреннего рассеяния. Тогда все минимальные пассивные системы (соответственно рассеяния либо сопротивления) $c$ п. $\phi . \Theta(z)$ унитарно подобнь тогда и только тогда, когда определена матрица внутреннего рассеяния $s_{\Theta}(z)$, т.е. когда $s_{\Theta}(\zeta)$ является радиальныцм граничным.м значением функции $s_{\Theta}(z)$ класса $\mathscr{S}\left(U_{0}, Y_{0}\right)$.

Teорема 3. Пусть $\Theta \in \mathscr{S}(U, Y)$ либо $\Theta \in \mathscr{C}(U)$. Пусть, кроме того, $s_{\Theta}(\zeta)\left(\in L^{\infty}\left(U_{0}, Y_{0}\right)\right)$ - соответствующий субоператор внутреннего рассеяния. Пусть $\Gamma_{\Theta}\left(\in\left[H^{2}\left(U_{0}\right), K^{2}\left(Y_{0}\right)\right]\right)$ - ганкелев оператор с символом $s_{\Theta}(\zeta)$. Тогда все минимальнье пассивнье системы (соответственно рассеяния или сопротивления) с п.. $\Theta(z)$ подобны тогда и только тогда, когда область значений оператора $\Gamma_{\Theta}$ замкнута в $K^{2}\left(Y_{0}\right)$. 
ДоКАЗАТЕЛЬСТво ТЕОРемЫ 2. Пусть $\Theta \in \mathscr{S}(U, Y)$ либо $\Theta \in \mathscr{C}(U)$.

Если $\varphi_{\Theta}=0$ либо $\psi_{\Theta}=0$, то по соглашению $s_{\Theta}(\zeta)=0$. Следовательно, $s_{\Theta}(\zeta) \equiv 0$. С другой стороны, согласно теореме 1 в рассматриваемом случае все минимальные пассивные системы с п.ф. $\Theta(z)$ унитарно подобны. Таким образом, утверждения теорем 2 и 3 справедливы в случае, когда $\varphi_{\Theta}=0$ либо $\psi_{\Theta}=0$. Пусть $\varphi_{\Theta} \neq 0$ и $\psi_{\Theta} \neq 0$. Рассмотрим простую консервативную систему (рассеяния либо сопротивления) $\Sigma=(A, B, C, D ; X, U, Y)$ с п.ф. $\Theta(z)$. Пусть $\Sigma_{0}=\Sigma_{\text {rest }, 1}=\left(A_{0}, B_{0}, C_{0}, D ; X_{0}, U, Y\right)$ и $\Sigma_{\bullet}=\Sigma_{\text {rest }, 2}=\left(A_{\bullet}, B \bullet, C \bullet, D ; X \bullet, U, Y\right)$ - первое и второе сужения системы $\Sigma$. Согласно предложению $3 \Sigma_{0}$ и $\Sigma$ • - минимальные оптимальная и $*$-оптимальная системы (рассеяния или сопротивления) с п.ф. $\Theta(z)$. Следовательно, они слабо подобны. Более того, замкнутый оператор $R$, осуществляющий слабое подобие систем $\Sigma_{\bullet}$ и $\Sigma_{0}$, действующий из $X_{\bullet}$ в $X_{0}$, действует на линеале

$$
\operatorname{Im}(\dot{A} / \dot{B})=\left\{\dot{x}=\sum_{0}^{n} \dot{A}^{k} \dot{B} u_{k}: n \geqslant 0, u_{k} \in U, 0 \leqslant k \leqslant n\right\}
$$

по формуле

$$
R\left(\sum_{0}^{n} A_{\bullet}^{k} B u_{k}\right)=\sum_{0}^{n} A_{0}^{k} B_{0} u_{k}
$$

Так как

$$
\left\|\sum_{0}^{n} A_{0}^{k} B_{0} u_{k}\right\| \leqslant\left\|\sum_{0}^{n} A_{\bullet}^{k} B \bullet u_{k}\right\|
$$

то $R$ - сжимающий оператор, определенный на всем пространстве $X$ •. Далее, так как

то получаем, что

$$
\begin{aligned}
\sum_{0}^{n} A_{\bullet}^{k} B \bullet u_{k} & =P_{X} \sum_{k=0}^{n} A^{k} B u_{k}, \\
\sum_{0}^{n} A_{0}^{k} B_{0} u_{k} & =P_{X_{0}} \sum_{k=0}^{n} A^{k} B u_{k},
\end{aligned}
$$

$$
R P_{X_{\bullet}} x=P_{X_{0}} x, \quad x \in X_{\Sigma}^{c} .
$$

Следовательно, учитывая, что $X \bullet \subset X_{\Sigma}^{c}$, получаем

$$
R x=P_{X_{0}} x, \quad x \in X_{\bullet} .
$$

Из этой формулы видно, что $R$ является унитарным отображением $X \bullet$ на $X_{0}$ тогда и только тогда, когда $X_{0}=X_{\bullet}$. Так как

$$
X_{0}=X \ominus\left(\mathfrak{D}_{+} \oplus\left(\mathfrak{D}_{-} \cap X_{\Sigma}^{o}\right)\right), \quad X_{\bullet}=X \ominus\left(\left(\mathfrak{D}_{+} \cap X_{\Sigma}^{c}\right) \oplus \mathfrak{D}_{-}\right),
$$

то $X_{0}=X_{\bullet}$ тогда и только тогда, когда

$$
\mathfrak{D}_{+} \oplus\left(\mathfrak{D}_{-} \cap X_{\Sigma}^{o}\right)=\left(\mathfrak{D}_{+} \cap X_{\Sigma}^{c}\right) \oplus \mathfrak{D}_{-},
$$

а это равенство имеет место тогда и только тогда, когда $\mathfrak{D}_{-}$и $\mathfrak{D}_{+}$ортогональны в $X$, где $\mathfrak{D}_{-}$и $\mathfrak{D}_{+}$определены в $(4)$. Остается вспомнить, что $\mathfrak{D}_{-}$и $\mathfrak{D}_{+}$ортогональны тогда и только тогда, когда $s_{\Theta}(\zeta)$ является радиальным граничным значением функции $s_{\Theta}(z) \in \mathscr{S}\left(U_{0}, Y_{0}\right)$. 
ДоКАЗАТЕЛЬСТВО ТЕОРЕМЫ 3. Как уже показано при доказательстве предыдушей теоремы, оператор $R\left(\in\left[X_{\bullet}, X_{0}\right]\right)$, определенный по формуле $(24)$, устанавливает слабое подобие систем $\Sigma_{\bullet}$ и $\Sigma_{0}$. Осталось выяснить, при каких условиях на $\Theta$ этот оператор ограниченно обратим. Ограниченная обратимость оператоpa $R$ равносильна ограниченной обратимости оператора

$$
R^{*}=P_{X_{\bullet}} \mid X_{0}\left(\in\left[X_{0}, X_{\bullet}\right]\right) \text {. }
$$

Этот оператор обратим, и по теореме Банаха он является ограниченно обратимым тогда и только тогда, когда его область значений замкнута, т.е. когда линеал

$$
\mathscr{L}=P_{X} X_{0}
$$

замкнут. Имеем

$$
\mathscr{L}=P_{X} P_{X_{\Sigma}^{c}} X_{0}=P_{X_{\bullet}} P_{X_{\Sigma}^{c}}\left(X_{\Sigma}^{o} \ominus\left(X_{\Sigma}^{o} \cap \mathfrak{D}_{-}\right)\right)=P_{X} P_{X_{\Sigma}^{c}} X_{\Sigma}^{o}=P_{X_{\Sigma}^{c}} X_{\Sigma}^{o}
$$

Итак, нужно выяснить, при каких условиях на $\Theta$ линеал

$$
\mathscr{L}=P_{X_{\Sigma}^{c}} X_{\Sigma}^{o}
$$

замкнут. Через $M^{\perp}$ обозначим ортогональное дополнение в $X$ к подпространству $M$. Имеем

$$
\left(X_{\Sigma}^{c}\right)^{\perp}=\mathfrak{D}_{-}, \quad\left(X_{\Sigma}^{o}\right)^{\perp}=\mathfrak{D}_{+} .
$$

Линеал $\mathscr{L}$ замкнут тогда и только тогда, когда замкнут линеал $\left(X_{\Sigma}^{c}\right)^{\perp}+X_{\Sigma}^{o}$, см. [21; лекция VIII, доказательство леммы о близких подпространствах]. Но

$$
\left(X_{\Sigma}^{c}\right)^{\perp}+X_{\Sigma}^{o}=\mathfrak{D}_{-}+\mathfrak{D}_{+}^{\perp}
$$

а линеал $\mathfrak{D}_{-}+\mathfrak{D}_{+}^{\perp}$ замкнут тогда и только тогда, когда замкнут линеал

$$
\mathscr{L}_{1}=P_{\mathfrak{D}_{+}} \mathfrak{D}_{-}
$$

Учитывая формулы (21)-(23), получаем:

$$
\mathscr{L}_{1}=\Gamma \mathfrak{D}_{-}=\mathscr{F}_{+}^{*} \Gamma_{\Theta} \mathscr{F}_{-} \mathfrak{D}_{-}=\mathscr{F}_{+}^{*} \Gamma_{\Theta} H^{2}\left(\mathfrak{N}_{-}\right)
$$

Так как $\mathscr{F}_{+}^{*}$ изометрически отображает $K^{2}\left(\mathfrak{N}_{-}\right)$на $\mathfrak{D}_{+}$, то линеал $\mathscr{L}_{1}$ замкнут тогда и только тогда, когда замкнут линеал

$$
\mathscr{L}_{2}=\Gamma_{\Theta} H^{2}\left(\mathfrak{N}_{-}\right) .
$$

Итак, доказано, что оператор $R\left(\in\left[X_{\bullet}, X_{0}\right]\right)$, осуществляющий слабое подобие систем $\Sigma_{\bullet}$ и $\Sigma_{0}$, является ограниченно обратимым тогда и только тогда, когда область значения ганкелева оператора $\Gamma_{\Theta}$ замкнута. 


\section{§5. Случай псевдопродолжимой передаточной функции}

Пусть теперь $\operatorname{dim} U+\operatorname{dim} Y<\infty$.

Мероморфная в $K$ функция $h(z)$ со значениями из $[U, Y]$ относится к классу Неванлинны $N(U, Y)$, если она представима в виде $h(z)=h_{2}^{-1}(z) h_{1}(z)$, где $h_{1} \in$ $\mathscr{S}(U, Y), h_{2}$-скалярная функция класса Шура $\mathscr{S}(=\mathscr{S}(\mathbb{C}, \mathbb{C}))$. Функция $h(z)$ класса $N(U, Y)$ относится к классу Смирнова $N_{+}(U, Y)$, если она представима в виде $h(z)=h_{2}^{-1}(z) h_{1}(z)$, где $h_{1} \in \mathscr{S}(U, Y), h_{2}$ - внешняя функция класса $\mathscr{S}$. Если $h(z) \in N_{+}(U, Y)$ и $h^{-1}(z) \in N_{+}(Y, U)$, то $h(z)$ относится к классу $N_{\text {out }}(U, Y)$ внешних функций из $N(U, Y)$. Имеем:

$$
\mathscr{S}(U, Y) \subset N_{+}(U, Y), \quad \mathscr{C}(U) \subset N_{+}(U, U)
$$

Более того, если $\Theta(z) \in \mathscr{C}(U)$ и $\operatorname{Re} \Theta(z)>0$ в $K$, то $\Theta^{-1}(z) \in \mathscr{C}(U)$ и потому $\Theta(z) \in N_{\text {out }}(U, U)$.

Говорят, что функция $h(z)$ класса $N(U, Y)$ имеет мероморфноепсевдопродолжение во внешность $K_{e}=\{z: 1<|z| \leqslant \infty\}$ круга $K$ с ограниченной характеристикой Неванлинны в $K_{e}$, если существует мероморфная в $K_{e}$ функция $h_{-}(z)$ такая, что $h_{-}(1 / z) \in N(U, Y)$ и

$$
\lim _{r \uparrow 1} h(r \zeta)=\lim _{r \downarrow 1} h_{-}(r \zeta) \text { п.в., }|\zeta|=1 .
$$

Это псевдопродолжение $h_{-}(z)$ также будем обозначать через $h(z)$, как и исходную функцию, и будем рассматривать $h(z)$ как мероморфную функцию в объединении $K \cup K_{e}=(C \cup\{\infty\}) \backslash \partial K$.

Через $\Pi(U, Y)$ обозначим класс функций $h(z)$ из $N(U, Y)$, имеюших меромор фное псевдопродолжение в $K_{e}$ с $h(1 / z) \in N(U, Y)$. Если $M \subset N(U, Y)$, то через П $M$ обозначим пересечение $M \cap \Pi(U, Y)$. Так,

$$
\Pi \mathscr{S}(U, Y)=\Pi(U, Y) \cap \mathscr{S}(U, Y), \quad \Pi \mathscr{C}(U)=\Pi(U, U) \cap \mathscr{C}(U) .
$$

Пусть $\Theta \in \Pi \mathscr{S}(U, Y)$. Тогда функции

$$
\Delta_{\Theta}(\zeta):=I-\Theta^{*}(\zeta) \Theta(\zeta) \geqslant 0 \text { и } \Delta_{\Theta^{*}}(\zeta):=I-\Theta(\zeta) \Theta^{*}(\zeta) \geqslant 0 \text { п.в., }|\zeta|=1,
$$

являются граничными значениями функций

$$
\Delta_{\Theta}(z):=I-\Theta^{*}\left(\frac{1}{\bar{z}}\right) \Theta(z) \text { и } \Delta_{\Theta}(z):=I-\Theta(z) \Theta^{*}\left(\frac{1}{\bar{z}}\right)
$$

классов $N(U, U)$ и $N(Y, Y)$ соответственно. По теореме Розенблюма-Ровняка [22] для $\Delta_{\Theta}(\zeta)$ и $\Delta_{\Theta}(\zeta)$ разрешимы факторизационные задачи а) и б) в (12) и максимальные решения $\varphi_{\Theta}$ и $\psi_{\Theta}$ этих задач удовлетворяют условиям

$$
\varphi_{\Theta} \in \Pi \mathscr{S}\left(U, Y_{0}\right), \quad \psi_{\Theta} \in \Pi \mathscr{S}\left(U_{0}, Y\right)
$$


Поэтому субоператор внутреннего рассеяния $s_{\Theta}(\zeta)$ определяется каждым из эквивалентных соотношений

$$
\begin{array}{ll}
\text { а) } s_{\Theta}(\zeta) \psi_{\Theta}^{*}(\zeta)=-\varphi_{\Theta}(\zeta) \Theta^{*}(\zeta) \text { п.в., }|\zeta|=1, \\
\text { б) } \varphi_{\Theta}^{*}(\zeta) s_{\Theta}(\zeta)=-\Theta^{*}(\zeta) \psi_{\Theta}(\zeta) \text { п.в., }|\zeta|=1,
\end{array}
$$

и $s_{\Theta}(\zeta)$ является граничньп значением некоторой функции $s_{\Theta}(z)$ класса $N\left(U_{0}, Y_{0}\right)$ (см. [14]), так что соотношения (26) эквивалентны соответствующим уравнениям для $s_{\Theta}(z)$ :

$$
\begin{aligned}
& \text { а) } s_{\Theta}(z) \psi_{\Theta}^{*}\left(\frac{1}{\bar{z}}\right)=\varphi_{\Theta}(z) \Theta^{*}\left(\frac{1}{\bar{z}}\right) \\
& \text { б) } \varphi_{\Theta}^{*}\left(\frac{1}{\bar{z}}\right) s_{\Theta}(z)=-\Theta^{*}\left(\frac{1}{\bar{z}}\right) \psi_{\Theta}(z) \text {. }
\end{aligned}
$$

Аналогичное утверждение справедливо и для $\Theta(z) \in \Pi \mathscr{C}(U)$. В этом случае $\Delta_{\Theta}(z)$ определяется формулой

$$
\Delta_{\Theta}(z)=\Theta(z)+\Theta^{*}\left(\frac{1}{\bar{z}}\right)(\in N(U, U))
$$

и для $\Delta_{\Theta}(\zeta)$ разрешимы факторизационные уравнения а) и б) в (16).

Максимальные решения $\varphi_{\Theta}(z)$ и $\psi_{\Theta}(z)$ этих уравнений удовлетворяют условиЯM

$$
\varphi_{\Theta} \in \Pi H^{2}\left(U, Y_{0}\right), \quad \psi_{\Theta} \in \Pi H^{2}\left(U_{0}, U\right)
$$

Субоператор внутреннего рассеяния $s_{\Theta}(\zeta)$ снова является радиальным граничньм значением некоторой функции $s_{\Theta}(z) \in N\left(U_{0}, Y_{0}\right)$. Эта функция определяется каждьм из эквивалентных соотношений

$$
\begin{aligned}
& \text { а) } s_{\Theta}(z) \psi_{\Theta}^{*}\left(\frac{1}{\bar{z}}\right)=\varphi_{\Theta}(z) \\
& \text { б) } \varphi_{\Theta}^{*}\left(\frac{1}{\bar{z}}\right) s_{\Theta}(z)=\psi_{\Theta}(z) .
\end{aligned}
$$

Итак, для $\Theta \in \Pi \mathscr{S}(U, Y)$ и для $\Theta \in \Pi \mathscr{C}(U)$ субоператор внутреннего рассеяния $s_{\Theta}(\zeta)$ является радиальным граничным значением некоторой функции $s_{\Theta}(z)$ класса $N\left(U_{0}, Y_{0}\right)$ и

$$
\left\|s_{\Theta}\right\|_{\infty}=\operatorname{ess} \sup \left\{\left\|s_{\Theta}(\zeta)\right\|:|\zeta|=1\right\} \leqslant 1
$$

Обозначим через $H^{\infty}(U, Y)$ класс голоморфных ограниченных в $K$ функций со значениями из $[U, Y]$,

$$
S_{\text {in }}(U)=\left\{s(z) \in \mathscr{S}(U, U): s^{*}(\zeta) s(\zeta)=I, s(\zeta) s^{*}(\zeta)=I \text { п.в., }|\zeta|=1\right\}
$$

- класс двусторонне внутренних функций $s(z)$ со значениями из $[U] ;$ для $a_{1}(z) \in$ $H^{\infty}\left(U_{1}, Y\right)$ и $a_{2}(z) \in H^{\infty}\left(U_{2}, Y\right)$ запись $\left(a_{1}, a_{2}\right)_{L}=I$ означает, что $a_{1}$ и $a_{2}$ взаимно просты слева, т.е. они не имеют общего непостоянного левого двусторонне 
внутреннего делителя; для $a_{1} \in H^{\infty}\left(U, Y_{1}\right)$ и $a_{2} \in H^{\infty}\left(U, Y_{2}\right)$ запись $\left(a_{1}, a_{2}\right)_{R}=I$ означает, что $a_{1}$ и $a_{2}$ взаимно просты справа.

Поскольку $s_{\Theta}(z) \in N\left(U_{0}, Y_{0}\right)$ и удовлетворяет условию $(28)$, то она в существенном однозначно представима в виде отношений
a) $s_{\Theta}(z)=b_{\bullet}^{-1}(z) s_{\bullet}(z)$,
б) $s_{\Theta}(z)=s_{0}(z) b_{0}^{-1}(z)$,

где

$$
\begin{array}{ll}
b_{\bullet} \in S_{\mathrm{in}}\left(Y_{0}\right), & s_{\bullet} \in \mathscr{S}\left(U_{0}, Y_{0}\right), \quad\left(b_{\bullet}, s_{\bullet}\right)_{L}=I, \\
b_{0} \in S_{\mathrm{in}}\left(U_{0}\right), & s_{0} \in \mathscr{S}\left(U_{0}, Y_{0}\right), \quad\left(b_{0}, s_{0}\right)_{R}=I,
\end{array}
$$

см., например, [23].

Функции $a_{1} \in H^{\infty}\left(U_{1}, Y\right)$ и $a_{2} \in H^{\infty}\left(U_{2}, Y\right)$ называются сильно взачмно простыми слева, если

$$
\inf \left\{\left\|a_{1}^{*}(z) y\right\|+\left\|a_{2}^{*}(z) y\right\|: z \in K, y \in Y,\|y\|=1\right\}>0
$$

В этом случае пишут $\left[a_{1}, a_{2}\right]_{L}=I$. Функции $a_{1} \in H^{\infty}\left(U, Y_{1}\right)$ и $a_{2} \in H^{\infty}\left(U, Y_{2}\right)$ называются сильно взаимно простыми справа, и в этом случае пишут $\left[a_{1}, a_{2}\right]_{R}=I$, если

$$
\inf \left\{\left\|a_{1}(z) u\right\|+\left\|a_{2}(z) u\right\|: z \in K, u \in U,\|u\|=1\right\}>0
$$

Пусть $H(b)=H^{2}(\mathscr{L}) \ominus b H^{2}(\mathscr{L})$ для $b \in S_{\text {in }}(\mathscr{L})$. По функции $s_{\Theta}(z)$, представленной в виде $(29)$, определим оператор $T_{\Theta}$ по формуле

$$
T_{\Theta}=M_{b_{\bullet}} \Gamma_{\Theta}\left|H\left(b_{0}\right)=P_{H\left(b_{\bullet}\right)} M_{s_{\bullet}}\right| H\left(b_{0}\right) .
$$

Teopema 4. Пyсmь $\Theta \in \Pi \mathscr{C}(U), \operatorname{dim} U<\infty(\Theta \in \Pi \mathscr{S}(U, Y), \operatorname{dim} U+$ $\operatorname{dim} Y<\infty)$. Пусть $s_{\Theta}(\zeta)$ - соответствующий субоператор внутреннего рассеяния. Пусть $b_{\bullet}, s_{\bullet}, b_{0}$ и $s_{0}$ взяты из представлений $s_{\Theta}(z)$ в (29). Пусть $\Gamma_{\Theta}$ - ганкелев оператор с символом $s_{\Theta}(\zeta)$. Пусть оператор $T_{\Theta}$ определен по формуле (31). Тогда следуюшие условия әквивалентны:

а) все минимальные пассивные системы сопротивления (соответственно рассеяния) с п.ф. $\Theta(z)$ подобны;

б) область значений ганкелева оператора $\Gamma_{\Theta}$ замкнута;

в) $\left[b_{\bullet}, s_{\bullet}\right]_{L}=I$;

г) $\left[b_{0}, s_{0}\right]_{R}=I$

д) оператор $T_{\Theta}$ ограниченно обратим либо $s_{\Theta}(z) \in \mathscr{S}\left(U_{0}, Y_{0}\right)$.

ДокАЗАТЕльство. Эквивалентность условий а) и б) установлена в теореме 3. Эквивалентность условий б), в) и г) установлена П. Фурманном для произвольной функции $s(z) \in N\left(U_{0}, Y_{0}\right)$.

Эквивалентность условий б) и д) проверяется непосредственно. 


\section{§6. Скалярный случай}

Остановимся теперь на случае, когда $\operatorname{dim} U=\operatorname{dim} Y=1$, т.е. п.ф. $\Theta(z)$ скалярная. В этом случае вместо обозначений $\mathscr{S}(U, Y), \mathscr{C}(U), N(U, Y), \Pi(U, Y)$ и т. д. будем писать $\mathscr{S}, \mathscr{C}, N$, П и т. д.

Пусть

$$
f_{\Theta}(\zeta)= \begin{cases}1-|\Theta(\zeta)|^{2}, & \text { если } \Theta \in \mathscr{S}, \\ 2 \operatorname{Re} \Theta(\zeta), & \text { если } \Theta \in \mathscr{C} .\end{cases}
$$

Факторизационное неравенство

$$
|\varphi(\zeta)|^{2} \leqslant f_{\Theta}(\zeta) \text { п.в., }|\zeta|=1,
$$

имеет ненулевое решение $\varphi \in H^{2}$ тогда и только тогда, когда

$$
\ln f_{\Theta} \in L^{1} .
$$

По теореме Сегё это имеет место тогда и только тогда, когда разрешимо факторизационное уравнение

$$
|\varphi(\zeta)|^{2}=f_{\Theta}(\zeta) \text { п.в., }|\zeta|=1, \quad \varphi \in H^{2} .
$$

Если это уравнение разрешимо, то $\varphi_{\Theta}(z)$, максимальное решение этого уравнения, - внешняя функция.

Субоператор внутреннего рассеяния $s_{\Theta}(\zeta)$ определяется по формуле

$$
\begin{aligned}
& s_{\Theta}(\zeta)=\frac{\varphi_{\Theta}(\zeta)}{\overline{\varphi_{\Theta}(\zeta)}}, \text { если } \Theta \in \mathscr{C}, \\
& s_{\Theta}(\zeta)=-\frac{\varphi_{\Theta}(\zeta)}{\overline{\varphi_{\Theta}(\zeta)}} \bar{\Theta}(\zeta), \quad \text { если } \Theta \in \mathscr{S} .
\end{aligned}
$$

Лемма 1. Пусть $\Theta \in \mathscr{S}$ либо $\Theta \in \mathscr{C}$. Пусть для $f=f_{\Theta}$ разрешимо факторизационное уравнение (34), т.е. выполняется условие (33). Тогда следующие условия эквивалентны:
a) $\Theta \in \Pi$,
б) $\varphi_{\Theta} \in \Pi$,
B) $s_{\Theta}(z) \in \Pi$
г) $s_{\Theta} \in N$.

ДоказАТЕЛЬСтво. Пусть $\Theta \in$ П. Тогда $f_{\Theta}(\zeta)$ - граничное значение функции $f_{\Theta}(z)$ класса П. Поэтому

$$
\overline{\varphi_{\Theta}(\zeta)}=\frac{f_{\Theta}(\zeta)}{\varphi_{\Theta}(\zeta)}
$$

- граничное значение функции класса $N$. Следовательно, $\varphi_{\Theta} \in$ П. Из формул $(35)$ и (36) вытекает, что при этом $s_{\Theta}(\zeta)$ является граничным значением некоторой функции $s_{\Theta}(z)$ класса П. Обратно, пусть $s_{\Theta}(\zeta)$ является граничным значением некоторой функции класса $N$. Пусть $\Theta \in \mathscr{S}$ и $\Theta \neq 0$. Тогда

$$
\Theta(\zeta) s_{\Theta}(\zeta)=-\frac{\varphi_{\Theta}^{2}(\zeta) \overline{\Theta(\zeta)} \Theta(\zeta)}{1-|\Theta(\zeta)|^{2}}=-\varphi_{\Theta}^{2}(\zeta)-\frac{\varphi_{\Theta}^{2}(\zeta)}{1-|\Theta(\zeta)|^{2}}
$$


- граничное значение функции класса $N$. Поэтому $f_{\Theta}(\zeta)=1-\overline{\Theta(\zeta)} \Theta(\zeta)-$ граничное значение функции класса $N$. Так как

$$
\overline{\Theta(\zeta)}=\frac{1-f_{\Theta}(\zeta)}{\Theta(\zeta)}
$$

то получаем, что $\Theta \in \Pi$.

Пусть $\Theta \in \mathscr{C}$ и $S_{\Theta}(z) \in N$. Тогда из формулы (34) вытекает, что $\varphi_{\Theta} \in \Pi$. Следовательно, $f_{\Theta}(\zeta)=\Theta(\zeta)+\Theta^{*}(\zeta)$ - граничное значение функции класса $N$, так что $\Theta \in \Pi$.

TeOpema 5. Пусть $\Theta \in \mathscr{C}($ (либо $\Theta \in \mathscr{S})$.

1. Пусть для функиии $f_{\Theta}$, определенной по формуле (32), не выполняется условие (33). Тогда все минимальные пассивнье системы сопротивления (рассеяния) с п.ф. $\Theta(z)$ унитарно подобны и консервативны.

2. Пусть для $f_{\Theta}$ выполняется условие (33), но $\Theta \notin$ П. Тогда минимальные оптимальная и *-оптимальная пассивнье системы сопротивления (рассеяния) $с$ п.ф. $\Theta(z)$ не подобны.

3. Пусть $\Theta \in \Pi \mathscr{C}(\Theta \in \Pi \mathscr{S})$ и $f_{\Theta}(\zeta)>0\left(\Theta \notin \mathscr{S}_{\text {in }}\right.$ соответственно $)$. Пусть $s_{\bullet}(z)$ и $b_{\bullet}(z)$ взяты из представления (29) с условием (30). Тогда:

а) все минимальные пассивные системы сопротивления (рассеяния) с n. $\phi . \Theta(z)$ унитарно подобны в том и только том случае, если $b_{\bullet}(z) \equiv$ const, m.е. если $s_{\Theta}(z) \in \mathscr{S}_{\text {in }}\left(s_{\Theta} \in \mathscr{S}\right.$ соответственно);

б) все минимальные пассивнье системы сопротивления (рассеяния) с n. $\phi . \Theta(z)$ подобны тогда и только тогда, когда $b_{\bullet}(z)$ и $s_{\bullet}(z)$ сильно взаимно просты, т.е. когда

$$
\inf \left\{\left|b_{\bullet}(z)\right|+\left|s_{\bullet}(z)\right|: z \in K\right\}>0 .
$$

ДокАЗАТЕЛЬСтво. Утверждение 1 теоремы вытекает из теоремы 1. Утверждение 3 а) вытекает из теоремы 2. Утверждение $3 б$ ) вытекает из теоремы 4.

Остается доказать утверждение 2 теоремы. Пусть $\Theta \notin$ П, но выполняется условие (32). По лемме $1 s_{\Theta}(\zeta)$ не является граничным значением никакой функции $s_{\Theta}(z)$ класса $N$. Тогда для ганкелева оператора $\Gamma_{\Theta}$ с символом $s_{\Theta}(\zeta)$ имеем

$$
\operatorname{Ker} \Gamma_{\Theta}=\{0\}
$$

Действительно, если

$$
\mathscr{L}=\operatorname{Ker} \Gamma_{\Theta}\left(\subset H^{2}\right) \neq\{0\},
$$

то $\mathscr{L}$, будучи инвариантным подпространством в $H^{2}$ относительно оператора $S_{+}$ умножения на $z$, представимо в виде $\mathscr{L}=b_{1} H^{2}$, где $b_{1} \in \mathscr{S}_{\text {in }}$. Тогда мы получили бы, что $s_{1}(\zeta):=s_{\Theta}(\zeta) b_{1}(\zeta)$ - граничное значение некоторой функции $s_{1}(z) \in \mathscr{S}$ и, следовательно, $s_{\Theta}(\zeta)$ - граничное значение функции класса $N$, что противоречит условию. При условии (37) область значений оператора $\Gamma_{\Theta}$ не может быть замкнутой. Действительно, в противном случае по теореме Банаха мы имели бы

$$
\left\|\Gamma_{\Theta} h\right\| \geqslant c\|h\|, \quad h \in H^{2},
$$


при некоторой константе $c$. Это невозможно, так как

$$
\lim _{n \rightarrow \infty}\left\|\Gamma_{\Theta} S_{+}^{n} h\right\|=0
$$

Остается применить теорему 3.

\section{Список литературы}

1. Аров Д. З. Пассивные линейные стационарные динамические системы // Сиб. матем. журн. 1979. Т. 20. № 2. С. 211-228.

2. Arov D.Z. A survey on passive networks and scattering systems which are lossless or have minimal losses // Archiv für Elektronik und Übertragungstechnik. Internat. J. Electronics Commun. 1995. V. 49. № 5/6. P. 252-265.

3. Arov D. Z. Passive linear systems and scattering theory // Dynamical systems, control, coding, computing vision. Basel: Birkhäuser, 1999. P. 27-44. (Progr. Systems Control Theory. V. 25.)

4. Лившии M. С. Операторы, колебания, волны. Открытые системы. М.: Наука, 1966.

5. Willems J. C. Dissipative dynamical systems. Part I: General theory; Part II: Linear systems with quadratic supply rates // Arch. Ration. Mech. Anal. 1973. V. 45. P. 321-393.

6. Hill D. J., Moylan P.J. Dissipative dynamical systems: basic input-output and state properties // J. Franklin Inst. 1980. V. 309. P. 327-357.

7. Helton $J . W$. The characteristic functions of operator theory and electrical network realization // Indiana Univ. Math. J. 1972. V. 22. P. 403-404.

8. Helton J. W. Discrete time systems, operator models, and scattering theory // J. Funct. Anal. 1974. V. 16. P. 15-38.

9. Калман Р., Фалб П., Арбиб М. Очерки по математической теории систем. М.: Мир, 1971.

10. Arov D. Z., Nudelman M. A. Passive linear stationary dynamical scattering systems with continuous time // Integral Equations Operator Theory. 1996. V. 24. P. 1-45.

11. Аров Д. З., Нудельман М. А. Критерий унитарного подобия минимальных пассивных систем рассеяния с заданной передаточной функцией // Укр. матем. журн. 2000. Т. 52. № 2. C. $147-156$.

12. Аров Д. З. Условия подобия всех минимальных пассивных систем рассеяния с заданной матрицей рассеяния // Функц. анализ и его прилож. 2000. Т. 34. № 4. С. 71-74.

13. Ball J. A., Cohen N. De Branges-Rovnyak operator models and systems theory: A survey // Oper. Theory Adv. Appl. 1991. V. 50. P. 93-136.

14. Аров Д. З. Устойчивые диссипативные линейные стационарные динамические системы рассеяния // J. Operator Theory. 1979. V. 1. P. 95-126.

15. Arov D. Z., Kaashoek M. A., Pik D. R. Minimal and optimal linear discrete time-invariant dissipative scattering systems // Integral Equations Operator Theory. 1997. V. 29. P. $127-154$.

16. Надь Б. С., Фояи Ч. Гармонический анализ операторов в гильбертовом пространстве. М.: Мир, 1970.

17. Аров Д. З. Оптимальные и устойчивые пассивные системы // Докл. АН СССР. 1979. T. 247. C. $265-268$.

18. Адамян В. М., Аров Д. З. Унитарные сцепления простых полуунитарных операторов // Математические исследования. Т. 1. № 2. Кишинев, 1966. С. 3-64.

19. Аров Д. З. Теория рассеяния с диссипацией энергии // Докл. АН СССР. 1974. Т. 216. № 4. C. $713-716$.

20. Boiko S.S., Dubovoj V.K. On some extremal problem connected with the suboperator of the scattering through inner channels of the system // Доповіді НАН Укр. 1997. Т. 4. C. $7-11$.

21. Никольский Н. К. Лекции об операторе сдвига. М.: Наука, 1980. 
22. Rosenblum M., Rovnyak J. Hardy classes and operator theory. New York: Oxford Univ. Press, 1985.

23. Fuhrmann P. A. Linear systems and operator in Hilbert space. New York: McGraw-Hill, 1981.

Южно- Украинский государственньй

педагогический университет им. К. Д. Ушинского,

г. Одесса
Поступила в редакцию 03.05.2001 